\title{
On Time-Frequency Synchronization in LoRa System: From Analysis to Near-Optimal Algorithm
}

This paper was downloaded from TechRxiv (https://www.techrxiv.org).

\section{LICENSE}

CC BY 4.0

SUBMISSION DATE / POSTED DATE

$12-01-2021 / 16-01-2021$

\section{CITATION}

Savaux, Vincent; Delacourt, Christophe; Savelli, Patrick (2021): On Time-Frequency Synchronization in LoRa System: From Analysis to Near-Optimal Algorithm. TechRxiv. Preprint. https://doi.org/10.36227/techrxiv.13560158.v1

$\mathrm{DOI}$

10.36227/techrxiv.13560158.v1 


\title{
On Time-Frequency Synchronization in LoRa System: From Analysis to Near-Optimal Algorithm
}

\author{
Vincent Savaux, Member, IEEE, Christophe Delacourt, and Patrick Savelli
}

\begin{abstract}
This paper deals with time and frequency synchronization in LoRa system based on the preamble symbols. A thorough analysis of the maximum likelihood (ML) estimator of the delay (time offset) and the frequency offset shows that the resulting cost function is not concave. As a consequence the $a$ priori solution to the maximization problem consists in exhaustively searching over all the possible values of both the delay and the frequency offset. Furthermore, it is shown that these parameters are intertwined and therefore they must be jointly estimated, leading to an extremely complex solution. Alternatively, we show that it is possible to recover the concavity of the cost function, from which we suggest a low-complexity synchronization algorithm, whose steps are described in detail. Simulations results show that the suggested method reaches the same performance as the ML exhaustive search, while the complexity is drastically reduced, allowing for a real-time implementation of a LoRa receiver.
\end{abstract}

Index Terms-LoRa, synchronization, maximum likelihood.

\section{INTRODUCTION}

In the recent years, the Internet of things (IoT) has enabled the connectivity of various devices, offering digital transformation across industry verticals [1]. Among the different technologies, the low power wide area networks (LPWANs) have been drawing much attention [2], [3], as they provide features such as high battery life, low data rate, long range, and low communication fees. These features offer a high potential for sensor data collection and applications in the areas of smart metering, smart cities, environmental monitoring, asset tracking and many more. Among the LPWA solutions, the LoRa (Long Range) system has emerged as one of the most deployed technology, using non licensed spectrum resources [4]. This technology, originally developed by Semtech, relies on the so-called chirp spread spectrum (CSS) modulation technique [5], [6]. The resulting waveform has been analyzed in the time and frequency domain in [7]. The CSS signal requires an accurate time and frequency synchronization at the receiver side in order to recover the transmitted information correctly [5]. The performance of LoRa modulation scheme in terms of symbol and bit error rate has been analyzed in [8]-[10].

The first reference to time-frequency synchronization for chirp signals has been patented in the late 1990s [11], i.e. well before the release of LoRa technology. Based on the discrete Fourier transform of the received signal, it is less complex than an estimator based on the cross-correlation. For this reason,

V. Savaux, C. Delacourt, and P. Savelli are with $\mathrm{b}<>$ com, Rennes, France. emails: \{firstname.name@b-com.com\}

Manuscript received 2021. it has been reused in most of the methods in the literature dealing with synchronization in LoRa [12]-[15]. In [12], [14], the authors suggest to sequentially estimate the integer and the fractional contributions of the time and frequency offsets, based on the whole LoRa preamble, whereas it is proposed in [15] to track the oscillator fluctuations by means of the demodulated symbols within a LoRa frame. To achieve a lowcomplexity algorithm, the authors in [14] suggest to process the received signal at Nyquist rate while [12] investigates the effect of the oversampling rate. Although the method in [14] is less complex, it may be limited when the signal to noise ratio $(\mathrm{SNR})$ is weak (e.g. $\leq 0 \mathrm{~dB})$ due to the Nyquist rate processing.

In this paper, we rather take advantage of the oversampled LoRa signal to improve the synchronization performance, and in the same time we focus on an algorithm that quickly converges to the optimal solution in order to reduce the complexity of the synchronization process. To this end, a detailed theoretical analysis of the behavior of the frequency domain maximum likelihood (ML) estimator of the delay and frequency offset is developed for oversampled LoRa signals, based on a limited number $K$ symbols of the preamble. Ideally, to reduce the complexity, $K$ is chosen as a power of 2 . To the best of our knowledge, such a study has not been proposed in the literature about LoRa signal [7], and is an original added value of the paper. Furthermore, we keep the analysis general enough so that it can be applied to any oversampling factor $\geq 2$ and any size of preamble.

From the theoretical analysis, we show that the a priori solution to the ML estimator consists in an exhaustive search over all the possible values of both the delay and the frequency offset, leading to an extremely complex synchronization process. This is mainly due to the intertwined unknown parameters, such as stated in [12]. However, based on the theoretical results, we also deduce a low complexity estimator that quickly converges to the optimal solution. To improve the reproducibility of our result, we provide all the detailed steps of the algorithm as well as the corresponding source code. Simulations results reveal that the suggested algorithm reaches the ML exhaustive search, while allowing for a possible real-time software-based synchronization and demodulation process, even in condition of large spreading factors (SF).

The rest of the paper is organized as follows: Section II presents the received LoRa signal and introduces the ML estimation method for both the delay and the frequency offset. Section III is dedicated to the theoretical analysis of the behavior of the ML algorithm, and a low complexity synchronization algorithm is provided in Section IV. Simulations 


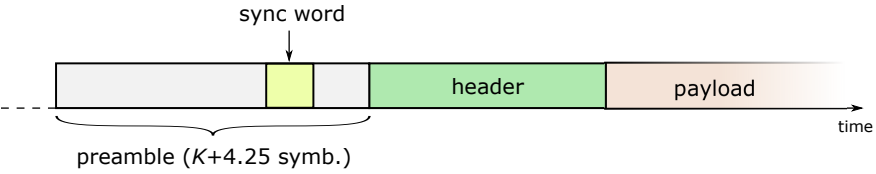

Fig. 1. A LoRa frame is composed of the preamble of $K+4.25$ symbols, the header, and the payload.

results are presented and discussed in Section V, and we draw our conclusions in Section VI.

Notations: Boldface letters a and normal font letters $a$ represent vectors and scalars, respectively. $\operatorname{DFT}(\mathbf{a})$ is the discrete Fourier transform (DFT) of the vector $\mathbf{a}, \mathbf{a} \circ \mathbf{b}$ is the Hadamard product of two vectors where $\mathbf{a}$ and $\mathbf{b}$ share the same size. Moreover, $\Re\{a\}$ gives the real part of a complex scalar $a$.

\section{Problem Statement}

This section deals with a detailed presentation of the LoRa CSS signal with a focus on the preamble. Moreover, basics on DFT-based ML synchronization are introduced as well. The LoRa modulation is based on the CSS, which is a constant modulus modulation therefore power efficient. Furthermore, the different spreading factors allow to adapt the robustness of the LoRa signal to the propagation conditions (path loss, interference, etc.) to the cost of a reduction of the data rate.

\section{A. System Model}

A LoRa frame consists in a preamble, a header, and a payload, as illustrated on the Fig. 1. In the following we focus on the preamble as it is used for detection and synchronization purposes, whereas the header and the payload carry binary information for control and data, respectively. The way the binary stream from medium access control (MAC) layer is modulated on chirps is out of the scope of this paper, but numerous papers (e.g. [7], [12], [16]) give details about the LoRa modulation. As depicted in Fig. 1, the preamble is composed of $K+4.25$ symbols, where $K$ can be set by the operator ( $K=8$ is usually the default value). The $K$ first symbols, denoted by $s_{i}(t)$ (in continuous time representation), $i=0,1, . ., K-1$ and $t \in\left[i T_{s},(i+1) T_{s}\right]$, are defined as

$$
s_{i}(t)=\exp \left(2 j \pi \frac{B_{w}\left(t-(2 i+1) T_{s}\right)}{2 T_{s}} \cdot t+j \phi\right),
$$

where $B_{w}$ is the bandwidth of LoRa signal, typically 125 $\mathrm{kHz}$ (but $250 \mathrm{kHz}$ and $500 \mathrm{kHz}$ are available as well), $T_{s}$ is the symbol duration such that $B_{w} T_{s}=2^{S F}$, and $\phi$ is a constant phase (usually omitted). The parameter $S F$ is called the spreading factor and is taken among $\{7,8, . ., 12\}$. The $K$ symbols are followed by two symbols that carry the "sync. word", corresponding to the network identifier. The remaining is composed of two inverted phase symbols (called downchirp compared to the $K$ first symbols called upchirp), and a guard period of one quarter symbol period.

In practice, a sampled version of $s_{i}(t)$ must be considered. Thus, we define the sampling duration as $\frac{T_{s}}{N_{R}}$ with $N_{R}=$ $R 2^{S F}, R$ being the oversampling rate. The sampled version

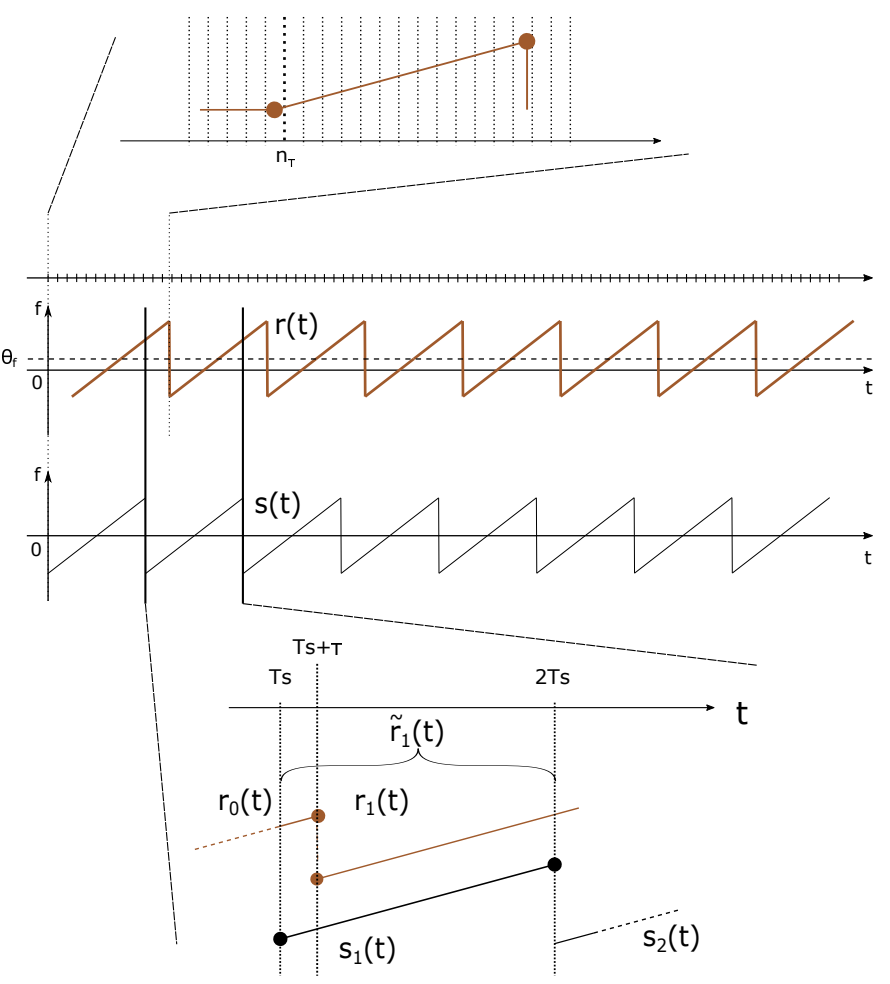

Fig. 2. System model: the first $K=8$ symbols of the preamble are represented. Center of the figure: the signal $r$ is received with a delay $\tau$ with respect to an arbitrary start time reference and a frequency offset $\theta_{f}$. Bottom: the signal $\tilde{r}_{i}$ is composed of the last samples of $r_{i-1}$ and the first samples of $r_{i}$. Top: the index of the first sample of $r[n]$ containing $s[n]$ is denoted $n_{\tau}$.

of $s_{i}(t)$ is obtained by substituting $t=n \frac{T_{s}}{N_{R}}$ in (1) with $n \in$ $\llbracket i N_{R},(i+1) N_{R}-1 \rrbracket$, hence leading to:

$$
s_{i}[n]=\exp \left(2 j \pi \frac{B_{w} T_{s}}{2}\left(\frac{n}{N_{R}}-(2 i+1)\right) \cdot \frac{n}{N_{R}}+j \phi\right) .
$$

In the following, we assume that the instant of detection of the LoRa frame is before the beginning of the frame and we arbitrarily set the reference start time $(t=0)$ between the instant of detection and the start of the received LoRa frame. Thus, the received signal is delayed by $\tau \geq 0$ compared with $t=0$. Moreover, the index of the first sample of the received frame is denoted by $n_{\tau}$, as illustrated in Fig. 2 . We assume that the LoRa signal bandwidth is narrow enough to consider a onetap block fading channel $h$ such that the received signal $r_{i}[n]$ can be expressed as in (3) where $w$ is the complex additive white Gaussian noise (AWGN) such that $w \sim \mathbb{C} \mathcal{N}\left(0, \sigma^{2}\right), \theta_{f}$ is the frequency offset between the transmitter and the receiver, and $\psi$ is an unknown additional phase.

Before tackling the time-frequency synchronization process in LoRa, we first introduce further notations related to the sampled time-frequency domain. To this end, we define the time sampling rate $R_{0} \leq R$ of the signals at the input of the DFT, and we define the ratio $\eta=\frac{R}{R_{0}}$. For instance, in the example in Fig. 3, we have $R_{0}=\frac{R}{2}$. Thus, the fine granularity offered by the oversampling rate $R$ ensures a good time precision to the synchronization over the time domain, whereas $R_{0} \leq R$ allows to reduce the complexity of the 


$$
r_{i}[n]=\left\{\begin{array}{l}
w[n], \text { if } n \in \llbracket 0, n_{\tau}-1 \rrbracket, \\
h \exp \left(2 j \pi \frac{B_{w} T_{s}}{2}\left(\frac{n}{N_{R}}-(2 i+1)-\frac{\tau}{T_{s}}\right)\left(\frac{n}{N_{R}}-\frac{\tau}{T_{s}}\right)+j \phi\right) \cdot \exp \left(2 j \pi \theta_{f} T_{s}\left(\frac{n}{N_{R}}-\frac{\tau}{T_{s}}\right)+j \psi\right)+w[n], \\
\quad \text { if } n \in \llbracket n_{\tau}+i N_{R}, n_{\tau}+(i+1) N_{R}-1 \rrbracket,
\end{array}\right.
$$
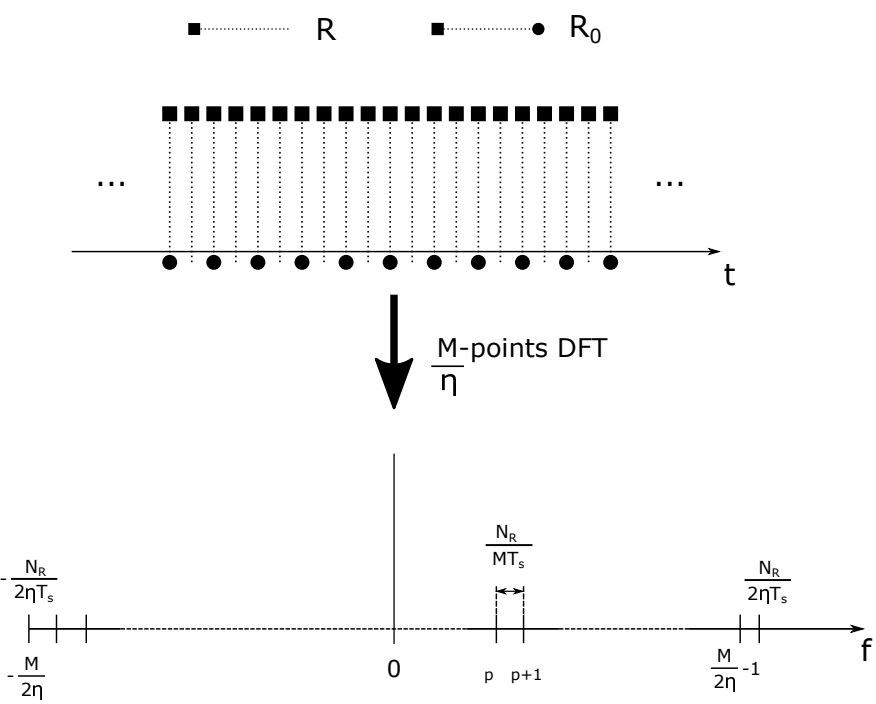

Fig. 3. Notations related to the sampling of the time and frequency domains.

synchronization process by reducing the size of the DFT, which is proportional to $R_{0}$.

For a matter of clarity, we denote by $M$ the size of the observation window (e.g. the length of the preamble) oversampled with rate $R$. All other sizes are related to the oversampled rate $R$ by using $\eta$. Thus, the DFT sizes are given by $\frac{M}{\eta}$. Furthermore, we will suppose that $\frac{M}{\eta}$ is evenly-valued, then the index $p$ of sampled frequency domain is such that $p \in\left\{-\frac{M}{2 \eta}, . ., 0, . ., \frac{M}{2 \eta}-1\right\}$, and the distance between two frequency samples $p$ and $p+1$ corresponds to a frequency spacing of $\frac{N_{R}}{M T_{s}}$.

\section{B. Synchronization}

This section is dedicated to the time and frequency synchronization process, which consists in estimating the best couple $\left(k^{*}, p^{*}\right)$ corresponding to the time-frequency indexes (at oversampling rate $R$ ) which are the closest to the values $\left(\tau, \theta_{f}\right)$. The ML estimation of the sampled delay $k$ and frequency offset $p$, with respect to unknown parameters $h$ and $\psi$, can be expressed as

$$
\left(\hat{k}^{*}, \hat{p}^{*}\right)=\underset{k, p}{\arg \max }\left|Y_{k, p}\right|^{2},
$$

with $Y_{k, p}$ the $p$-th element of the vector defined as

$$
\mathbf{Y}_{k}=\operatorname{DFT}\left(\mathbf{r}_{k}^{R_{0}} \circ\left(\mathbf{s}^{R_{0}}\right)^{*}\right),
$$

where $\mathbf{r}_{k}^{R_{0}}$ and $\mathbf{s}^{R_{0}}$ are the vectors of size $\frac{M}{\eta} \times 1$ containing the samples $\{r[\eta n+k]\}$ and $\left\{s[\eta n]^{*}\right\}$, respectively, with $n \in$ $\llbracket 0, \frac{M}{\eta}-1 \rrbracket$. Thus, the input of the DFT is sampled at rate $R_{0}$, highlighted by $\eta n$. For $p \in\left\{-\frac{M}{2 \eta}, . ., 0, . ., \frac{M}{2 \eta}-1\right\}$ and given $k$,
$\left|Y_{k, p}\right|^{2}$ is called the periodogram of the time-shifted received signal multiplied by the conjugate reference preamble. The proof that the ML estimator of $\left(k^{*}, p^{*}\right)$ can be written through the simple expression in (4) is proved in Appendix A.

For further analysis of the ML estimator of $\left(k^{*}, p^{*}\right)$, we define the function $g[k]$

$$
g[k]=\max _{p}\left|Y_{k, p}\right|^{2},
$$

where, for a given $k$, finding the maximum of $\left|Y_{k, p}\right|^{2}$ with respect to $p$ is straightforward. It should be noted that the solving of (4) should be a priori carried out only through an exhaustive search over the parameters $k$ and $p$. This assumption will be verified in Section III thanks to the behavior analysis of $g[k]$, and a solution to reduce the complexity of the exhaustive search will be suggested afterwards.

\section{BEHAVIOR ANALYSIS}

\section{A. Behavior of $g[k]$}

In this section, we assume that $\tau<<T_{s}$ and $R_{0}>1$, before generalizing to any $\tau$ value afterwards. Note that the case $R_{0}=1$ corresponding to the Nyquist sampling rate at the input of the DFT is not tackled in this paper as it involves specific processes. Furthermore, we limit the size of the observation window to the $K$ first symbols of the preamble. The reasons behind this choice are twofold: i) the sync word (carrying the network identifier) is generally unknown and cannot then be used for synchronization, and ii) $K$ is usually set to $K=8$, allowing for the use of fast Fourier transform (FFT) instead of DFT, therefore simplifying the synchronization process.

To analyze the behavior of $g[k]$, we define $\tilde{r}_{i}[n-k]$ as in (7). Thus, $\tilde{r}_{i}[n+k]$ consists of the $n_{\tau}-k$ last samples of $r_{i-1}$ (corresponding to noise samples if $i=0$ ), and the $N_{R}-n_{\tau}+k$ first samples of $r_{i}$, such as illustrated in Fig. 2. It is shown in Appendix B that $\left|Y_{k, p}\right|^{2}$ can be expressed as follows in (8), where $A_{1}=\left\lceil\left(n_{\tau}-k\right) / \eta\right\rceil$ and $A_{2}=\left(N_{R} / \eta-\left\lceil\left(n_{\tau}-k\right) / \eta\right\rceil\right)$, and where $\operatorname{asinc}_{\gamma_{i}}(),. i \in\{1,2\}$, is the aliased sinc function defined as

$$
\operatorname{asinc}_{\gamma_{i}}\left(A_{i}\right)=\frac{\sin \left(\pi \eta \gamma_{i}(k, p) A_{i}\right)}{A_{i} \sin \left(\pi \eta \gamma_{i}(k, p)\right)},
$$

in which $\gamma_{1}(k, p)$ and $\gamma_{2}(k, p)$ are given by:

$$
\gamma_{1}(k, p)=B_{w} T_{s}\left(\frac{1}{N_{R}}+\frac{k}{N_{R}^{2}}-\frac{\tau}{T_{s} N_{R}}\right)+\frac{\theta_{f} T_{s}}{N_{R}}-\frac{p}{M} .
$$

and

$$
\gamma_{2}(k, p)=B_{w} T_{s}\left(\frac{k}{N_{R}^{2}}-\frac{\tau}{T_{s} N_{R}}\right)+\frac{\theta_{f} T_{s}}{N_{R}}-\frac{p}{M} .
$$

Despite its apparent complexity, (8) can be easily interpretable, and can be even used to develop a low-complexity 


$$
\tilde{r}_{i}[n+k]=\left\{\begin{array}{l}
w[n], \text { if } n \in \llbracket 0, n_{\tau}-k-1 \rrbracket \\
h \exp \left(2 j \pi \frac{B_{w} T_{s}}{2}\left(\frac{(n+k)}{N_{R}}-(2 i-1)-\frac{\tau}{T_{s}}\right)\left(\frac{n+k}{N_{R}}-\frac{\tau}{T_{s}}\right)+j \phi\right) \cdot \exp \left(2 j \pi \theta_{f} T_{s}\left(\frac{n+k}{N_{R}}-\frac{\tau}{T_{s}}\right)+j \psi\right)+w[n], \\
\quad \text { if } n \in \llbracket i N_{R}, n_{\tau}+i N_{R}-k-1 \rrbracket, \text { and } i>0 \\
h \exp \left(2 j \pi \frac{B_{w} T_{s}}{2}\left(\frac{(n+k)}{N_{R}}-(2 i+1)-\frac{\tau}{T_{s}}\right)\left(\frac{n+k}{N_{R}}-\frac{\tau}{T_{s}}\right)+j \phi\right) \cdot \exp \left(2 j \pi \theta_{f} T_{s}\left(\frac{n+k}{N_{R}}-\frac{\tau}{T_{s}}\right)+j \psi\right)+w[n], \\
\quad \text { if } n \in \llbracket n_{\tau}-k+i N_{R},(i+1) N_{R}-1 \rrbracket,
\end{array}\right.
$$

$$
\begin{aligned}
\left|Y_{k, p}\right|^{2} & =\sum_{i=0}^{K-1} \sum_{n=i N_{R} / \eta}^{(i+1) N_{R} / \eta-1} \tilde{r}_{i}[\eta n+k] s_{i}[\eta n]^{*} e^{-2 j \pi \frac{p \eta n}{M}} \\
& =|h|^{2}\left|e^{j \pi \eta \gamma_{1}(k, p)\left(A_{1}-1\right)} A_{1} a \sin c_{\gamma_{1}}\left(A_{1}\right)+e^{2 j \pi B_{w} T_{s}\left(\frac{\tau}{T_{s}}-\frac{k}{N_{R}}\right)} e^{j \pi \eta \gamma_{2}(k, p)\left(N_{R} / \eta-1\right)} A_{2} a \operatorname{sinc} c_{\gamma_{2}}\left(A_{2}\right)\right|^{2} \\
& \times\left|\sum_{i=0}^{K-1} \exp \left(\frac{-2 j \pi p i}{K}+2 j \pi i T_{s} \theta_{f}\right)\right|^{2} .
\end{aligned}
$$

synchronization algorithm, such as suggested afterwards. In fact, the two functions asinc are the DFT of sinusoids corresponding to the cases where $r_{i-1}$ and $s_{i}$ overlap on one hand, and where $r_{i}$ and $s_{i}$ overlap on the other hand (see Fig. 2 ). Thus, for any $k$, the function $\operatorname{asinc}_{\gamma_{1}}\left(A_{1}\right)$ is maximum when $\gamma_{1}(k, p)$ is minimum, i.e. when $p$ takes the value $p_{1}$, according to (10):

$$
p_{1}=\left\lceil B_{w} T_{s}\left(K+\frac{k K}{N_{R}}-\frac{\tau K}{T_{s}}\right)+K \theta_{f} T_{s}\right\rfloor
$$

It must be emphasized that the delay and the frequency offset are intertwined, as both have an effect on the value of $p_{1}$. Similarly to (12), we find that for any $k$, the function asinc $_{\gamma_{2}}\left(A_{2}\right)$ is maximum when $\gamma_{2}(k, p)$ is minimum, i.e. when $p$ takes the value $p_{2}$, according to (11):

$$
p_{2}=\left\lceil B_{w} T_{s}\left(\frac{k K}{N_{R}}-\frac{\tau K}{T_{s}}\right)+K \theta_{f} T_{s}\right\rfloor .
$$

It can be noticed that $p_{1}-p_{2}=B_{w} T_{s} K=2^{S F} K$ corresponding to a frequency distance of $B_{w}$ between the two peaks, which is consistent with the theory. Fig. 4 shows the behaviors of the functions $A_{1} \operatorname{asinc}_{\gamma_{1}}\left(A_{1}\right)$ and $A_{2} \operatorname{asinc}_{\gamma_{2}}\left(A_{2}\right)$ versus $p$, where we considered the following parameters: $S F=7, K=8, B_{w}=250 \mathrm{kHz}, R=8, R_{0}=2$ (then $\eta=4), \tau=\frac{T_{s}}{4}, k=0, \theta_{f}=10 \mathrm{kHz}$. Such as indicated in Fig. 4, with the aforementioned parameters we find that $p_{2}=-297$ and $p_{1}=727$ such that $p_{1}-p_{2}=1024=8.2^{7}$. Furthermore, since $k=0$ the amplitudes of the functions are $A_{1}=\left\lceil R \tau B_{w} / \eta\right\rceil=64$ and $A_{2}=192$. Moreover, for any value $\theta_{f}$, one function is maximum for $k=n_{\tau}$ (corresponding to the best possible time synchronization) while the other one vanished.

It must be emphasized that, in the range of indexes $p$ close to $p_{1}$ and $p_{2}$, the overlap of one function (say $A_{1} a \sin c_{\gamma_{1}}$ ) over the other one $\left(A_{2} a \operatorname{sinc}_{\gamma_{2}}\right)$ can be neglected. Then we can assume the approximation in (14), which is much simpler than the expression in (8) as the phase terms can be omitted.

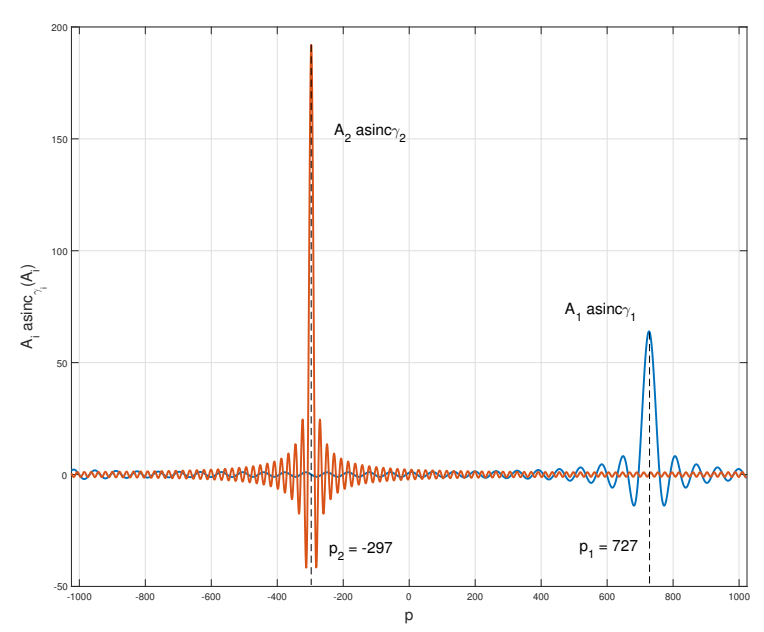

Fig. 4. asinc $_{\gamma}$ functions versus $p$, using $S F=7, K=8, B_{w}=250 \mathrm{kHz}$, $R=8, R_{0}=2$ (then $\left.\eta=4\right), \tau=\frac{T_{s}}{4}, k=0, \theta_{f}=10 \mathrm{kHz}$.

It remains to analyze the behavior of the last term involving the sum in (14), that we denote by $\mathcal{Q}$ for a matter of clarity.

For a given value of $\left(\tau, \theta_{f}\right)$, the function $\mathcal{Q}$ only depends on $k$, since $p$ also depends on $k$ from (12) and (13). Furthermore, it must be noted that $|\mathcal{Q}|$ can be bounded as

$$
0 \leq|\mathcal{Q}| \leq K
$$

where $|\mathcal{Q}|=0$ corresponds to the case where $Q$ is the sum of the $K k$-th roots of the unity, i.e. $\left(\frac{-2 j \pi p i}{K}+2 j \pi i T_{s} \theta_{f}\right) \propto \frac{2 j \pi i}{K}$. Conversely, the case $|\mathcal{Q}|=K$ corresponds to the case where $\left(\frac{-2 j \pi p i}{K}+2 j \pi i T_{s} \theta_{f}\right) \propto 2 j \pi$.

Fig. 5-(a) shows the behavior of $\mathcal{Q}$ versus $k$ for $p=p_{2}$, where $S F=7, K=8, B_{w}=250 \mathrm{kHz}, R=8, R=2$ (then $\eta=4$ ), $\tau=\frac{T_{s}}{10}$, and $\theta_{f}=700 \mathrm{~Hz}$. It can be seen that $\mathcal{Q}$ oscillates between maxima (close to eight) and minima (close to zero), and the maxima are reached every 


$$
g[k]=\max _{p}\left(\left|A_{1} \operatorname{asinc}_{\gamma_{1}}\left(A_{1}\right)\right|^{2}+\left|A_{2} \operatorname{asinc}_{\gamma_{2}}\left(A_{2}\right)\right|^{2}\right) \underbrace{\left|\sum_{i=0}^{K-1} \exp \left(\frac{-2 j \pi p i}{K}+2 j \pi i T_{s} \theta_{f}\right)\right|^{2}}_{\mathcal{Q}[k]},
$$

$k=K=8$ samples. Thus, the oscillations are due to the phase components $\frac{-2 j \pi p i}{K}$, while the value of the maximum and minimum of $\mathcal{Q}$ depends on $2 j \pi i T_{s} \theta_{f}$. In Fig. 5-(b) $g[k]$ is depicted with the same parameters as in Fig. 5-(a). We observe that $g[k]$ should reach its maximum with respect to $k$ if both $\mid A_{2}$ asinc $\left._{\gamma_{2}}\left(A_{2}\right)\right|^{2}$ and $|\mathcal{Q}|^{2}$ reach their respective maximum. Furthermore, since $|\mathcal{Q}|^{2}$ is not concave, then neither $g[k]$ is, hence no simple algorithm can be used to reach the maximum of $g[k]$. As a consequence, the only solution to solve (4) is a priori the exhaustive search, which is computationally complex.

However, in the following, we show that it is possible to recover the "envelop" of $g[k]$, which is almost concave. The "envelop" of $g[k]$, denoted by $g^{\prime}[k]$, corresponds to

$$
\begin{aligned}
g^{\prime}[k]= & \max _{p}\left(\left|A_{1} a_{p} \operatorname{sinc} \gamma_{\gamma_{1}}\left(A_{1}\right)\right|^{2}+\left|A_{2} \operatorname{asinc}_{\gamma_{2}}\left(A_{2}\right)\right|^{2}\right) \\
& \times\left|\sum_{i=0}^{K-1} \exp \left(2 j \pi i T_{s} \theta_{f}\right)\right|^{2} .
\end{aligned}
$$

It cannot be strictly concave in a general case due to the ceil operator $\lceil$.$\rceil in A_{1}$ and $A_{2}$, but we assume the shortcut regarding the concavity of the "envelop" of $g[k]$, which is illustrated in Fig.5-(b).

\section{B. Recovering Concavity through $g^{\prime}[k]$}

Although the phase component $2 j \pi i T_{s} \theta_{f}$ in $\mathcal{Q}$ is inherent to the system and then not controllable, the phase component $\frac{-2 j \pi p i}{K}$ depends on the designed preamble through the variable $K$. It is therefore possible to act on this component to avoid the variations of $g[k]$. We base our solution on Proposition 1 .

Proposition 1. $\forall p \in\left\{-\frac{M}{2 \eta}, . ., 0, . ., \frac{M}{2 \eta}-1\right\}$ and $\forall i \in$ $\llbracket 0, . ., K-1 \rrbracket, \exists q \in \llbracket 0, . ., K-1 \rrbracket$ such that

$$
\exp \left(\frac{-2 j \pi(p+q) i}{K}\right)=1
$$

Proof. $\forall p \in\left\{-\frac{M}{2 \eta}, . ., 0, . ., \frac{M}{2 \eta}-1\right\}, p$ can be rewritten $p=$ $a K+b$, where $a \in \mathbb{Z}$ and $b \in \llbracket 0, . ., K-1 \rrbracket$. Then we have

$$
\exp \left(\frac{-2 j \pi p i}{K}\right)=\exp \left(\frac{-2 j \pi b i}{K}\right) \text {. }
$$

Hence, by setting $q=K-1-b$, we obtain (17) where $q \in$ $\llbracket 0, . ., K-1 \rrbracket$, which concludes the proof.

Since Proposition 1 holds for any $p$ then it holds for $p=p_{1}$ or $p=p_{2}$. The suggested solution to recover the concavity of $g$ then consists in substituting (4) by

$$
\left(\hat{k}^{*}, \hat{p}^{*}\right)=\underset{k, p, q}{\arg \max }\left|Y_{k, p}^{q}\right|^{2},
$$

where $q \in \llbracket 0, . ., K-1 \rrbracket$ and

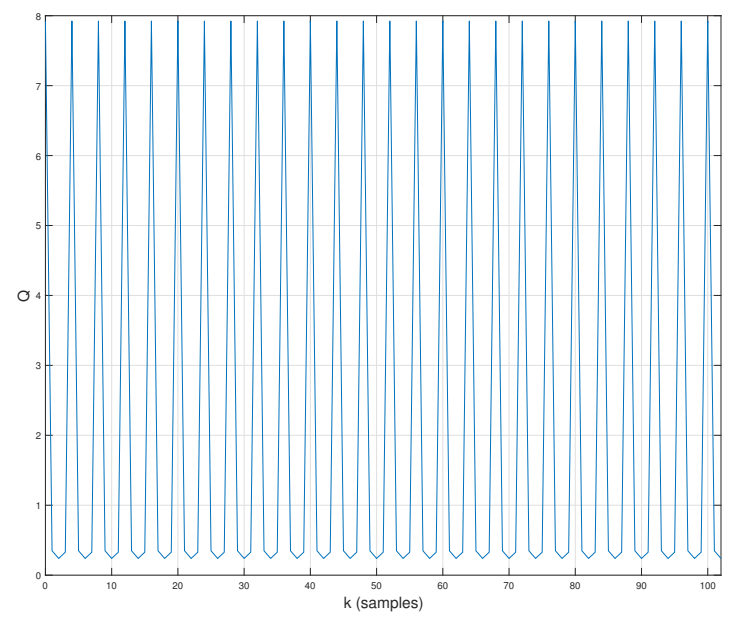

(a) $\mathcal{Q}$ versus samples $k$, for $\tau=\frac{T_{s}}{10}$ and $k \in\left\{0, n_{\tau}\right\}$.

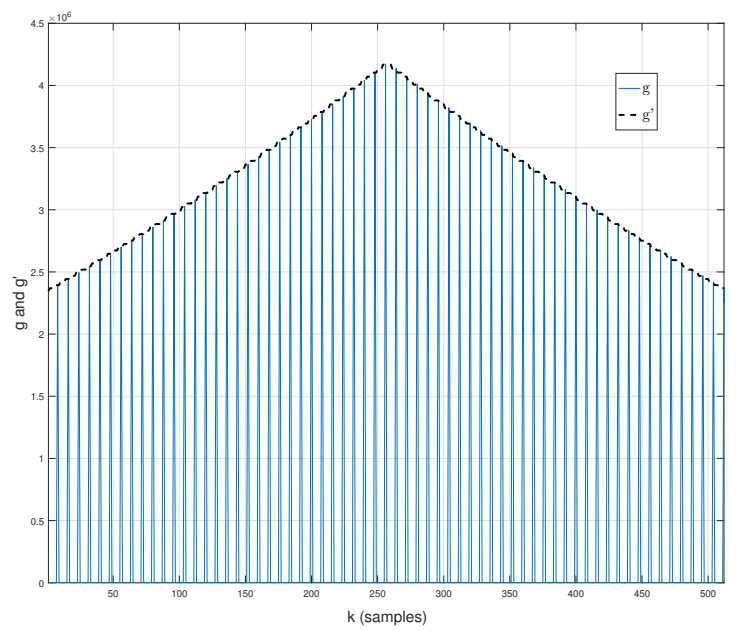

(b) $g$ and $g^{\prime}$ versus samples $k$, for $\tau=\frac{T_{s}}{10}$ and $k \in\left\{0,2 n_{\tau}\right\}$.

Fig. 5. $\mathcal{Q}$ versus samples $k$ (a), and $g[k]$ and $g^{\prime}[k]$ versus samples $k$ (b), using $S F=7, K=8, B_{w}=250 \mathrm{kHz}, R=8, R_{0}=2$ (then $\eta=4$ ), $p=p_{2}, \theta_{f}=830 \mathrm{~Hz}$.

$$
Y_{k, p}^{q}=\sum_{n=0}^{M / \eta-1} r[\eta n+k]\left(s[\eta n]^{*} e^{-2 j \pi \frac{q \eta n}{M}}\right) e^{-2 j \pi \frac{p \eta n}{M}},
$$

i.e. $\left|Y_{k, p}^{q}\right|$ is $p$-th component of the periodogram of the received signal multiplied by $s[\eta n]^{*} e^{-2 j \pi \frac{q \eta n}{M}}$. Thus, we redefine $g^{\prime}$ as

$$
g^{\prime}[k]=\max _{p, q}\left|Y_{k, p}^{q}\right|^{2},
$$

which exactly corresponds to $g^{\prime}$ in (16), and we show the 
behavior of $g^{\prime}$ in Fig. 5-(b). We observe that $g^{\prime}$ is indeed the "envelop" of $g$. We can then use the concavity property of $g^{\prime}$ to suggest a simple algorithm of synchronization in the next section.

Before dealing with the suggested synchronization algorithm, it must be emphasized that the behavior analysis of $g$ and $g^{\prime}$ can be easily extended to any $\tau$ value, by avoiding the assumption $\left(\tau<<T_{s}\right)$ that we made to obtain (35). In particular, it can be noted that if $\tau=u . T_{s}, u=0,1, . ., K-1$ and $k=0$, then $0 \leq|\mathcal{Q}| \leq K-u$. The same results is obtained when, for instance $\tau=(u+1) \cdot T_{s}, u=0,1, . ., K-1$ and $k=N_{R}$. This highlights the fact that the maximum value of $g$ is proportional to length of the overlapping window between $\mathbf{r}_{k}^{R_{0}}$ and $\mathbf{s}^{R_{0}}$ in (5). It results that $g^{\prime}$ oscillates similarly to the behavior shown in Fig. 5-(b) but between local maxima whose values are (inferiorly) proportional to $K-u$, and with a periodicity of $T_{s}$ (or equivalently $N_{R}$ samples). The behavior of $g$ can then be generally summarized for any $0 \leq \tau \leq K T_{s}$ as follows: $g$ oscillates between local maxima with a periodicity of $K$ samples (see Fig. 5-(a)), and the envelope $g^{\prime}$ of the oscillations itself oscillates between local maximal values with a periodicity of $N_{R}$ samples.

\section{LOW-COMPlEXITY Algorithm}

In this section, we describe both the exhaustive search and the proposed algorithm in pseudo code format. The aim is to make the processes easy to understand and the results easily reproducible. We use some support functions defined as:

- $\operatorname{len}(\mathbf{v})$ : returns the length of the vector $\mathbf{v}$,

- $\max (\mathbf{v})$ : returns the maximum value of the vector $\mathbf{v}$,

- $\operatorname{argmax}(\mathbf{v})$ : returns the index of the maximum value of the vector $\mathbf{v}$,

- $\operatorname{DFT}(\mathbf{v})$ : computes the Discrete Fourier Transform of the input vector $\mathbf{v}$.

Array slices are defined as $\mathbf{v}[$ start : stop : stride] and return a vector containing elements $\mathbf{v}[i]$ where $i=s t a r t+k *$ stride with $i<$ stop, and $k$ being a positive integer.

\section{A. Exhaustive synchronization algorithm}

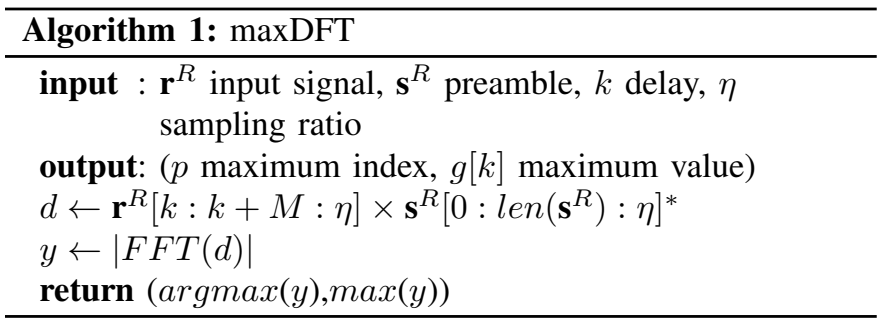

We introduce a first algorithm, $\max D F T$ (see Algorithm 1) which is the base operation for exhaustiveSyncro, as well as phase 1 , phase 2 and phase 3 used in the suggested method. It returns the maximum index and value of $\left|D F T\left(\mathbf{r}_{k}^{R_{0}} \circ\left(\mathbf{s}^{R_{0}}\right)^{*}\right)\right|$ in (5) for a fixed $k$. The sampling factor ratio $\eta$ is also provided to reduce the overall computational complexity which is similar to the DFT complexity: $O\left(\frac{M}{\eta} \log \left(\frac{M}{\eta}\right)\right)$.

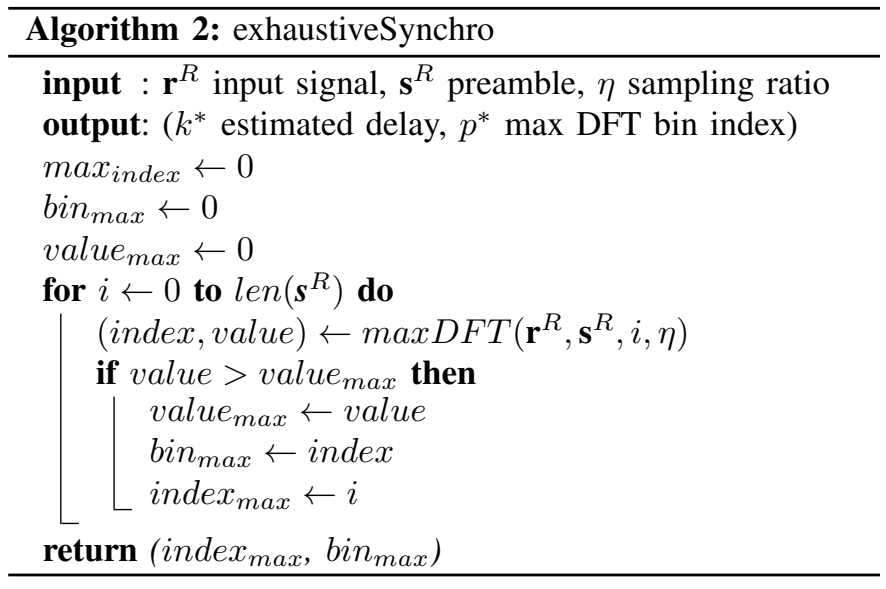

The exhaustive algorithm exhaustiveSynchro (see Algorithm 2) is a numerical translation of the exhaustive search in (4). The estimated frequency offset can then be obtained by matching the obtained bin index $p^{*}$ to the corresponding frequency of the DFT coefficients, but it is not further detailed as it is straightforward. The algorithm complexity is $O\left(\frac{M^{2}}{\eta} \log \left(\frac{M}{\eta}\right)\right)$ (corresponding to $M$ times $\max D F T$ ) which is computationally too expensive, especially for large SF values. Nevertheless this algorithms is used as a reference in simulation and it is also a good starting point for the understanding of the proposed algorithm.

\section{B. Low complexity synchronization algorithm}

Such as aforementioned, the aim of the suggested algorithm is to achieve the same performance as the exhaustive search by drastically reducing the computational complexity. This can be performed by recovering the concave envelop $g^{\prime}$ of $g$ such as in (21). Thus, the proposed algorithm is decomposed in 3 phases depicted in the remaining of this section.

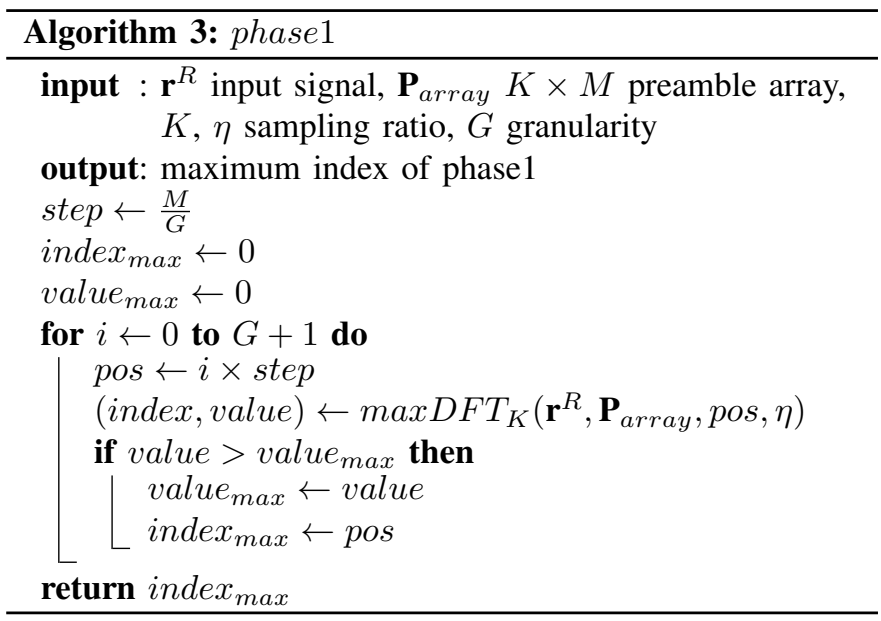

The first phase phase1 (see Algorithm 3) aims at finding an index $k_{0}$ such that $k_{0} \in \llbracket k^{*}-\frac{N_{R}}{2}, k^{*}+\frac{N_{R}}{2} \rrbracket$, therefore avoiding the convergence of the algorithm to a local maximum of $g^{\prime}$. In this phase we introduce the parameter $G$ that we call granularity in the following. From $G$ we define the subset $\Omega_{G}$ 
of $\llbracket 0, M-1 \rrbracket$ composed of evenly distributed time indexes $\Omega_{G}=\left\{k \mid k=0, \frac{M}{G}-1,2 \frac{M}{G}-1, . ., M-1\right\}$. Thus, the step phase 1 evaluates the "envelop" $g^{\prime}$ using (21) over the subset $\Omega_{G}$, and returns the index $k_{0}$ corresponding to the maximum of $g^{\prime}[k], k \in \Omega_{G}$. The index $k^{*}$ of (4) must then be located in a range of $\pm \frac{M}{2 G}$ samples from $k_{0}$. To ensure this, $G$ must satisfies $G \geq 2 K$. Note that $\mathbf{P}_{\text {array }}$ is the $K \times M$ array containing the $K$ versions of the preamble, i.e. $\mathbf{P}_{\text {array }}[q, n]=s[\eta n]^{*} e^{-2 j \pi \frac{q \eta n}{M}}$ such as used in (20). The complexity of this step is $O\left(G \frac{K M}{\eta} \log \left(\frac{M}{\eta}\right)\right)$. The function $\max D F T_{K}$ used in Algorithm 3 is hereby described.

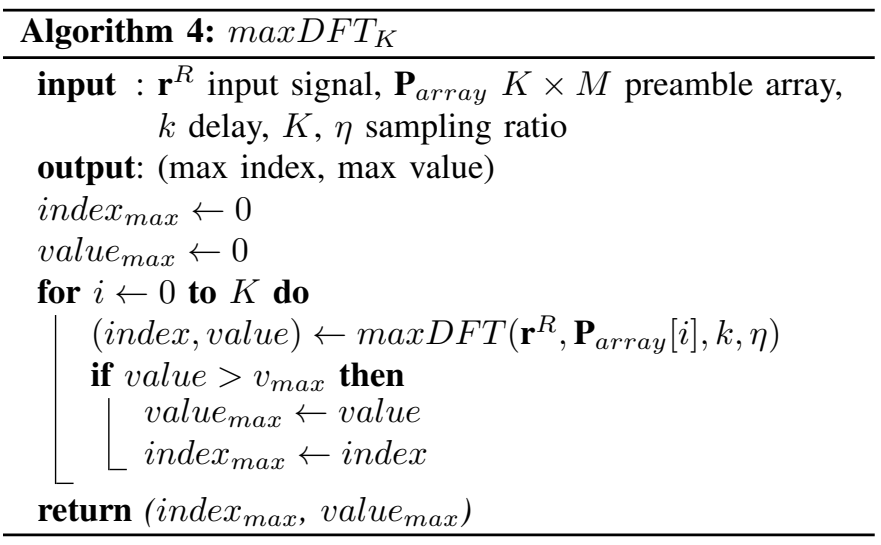

The function $\max D F T_{K}$ evaluates the envelop $g^{\prime}$ of $g$ (see (21)) for a certain $k$, such as detailed in Algorithm 4. Note that $\mathbf{P}_{\text {array }}[i]$ corresponds to the $i$-th row of $\mathbf{P}_{\text {array }}$.

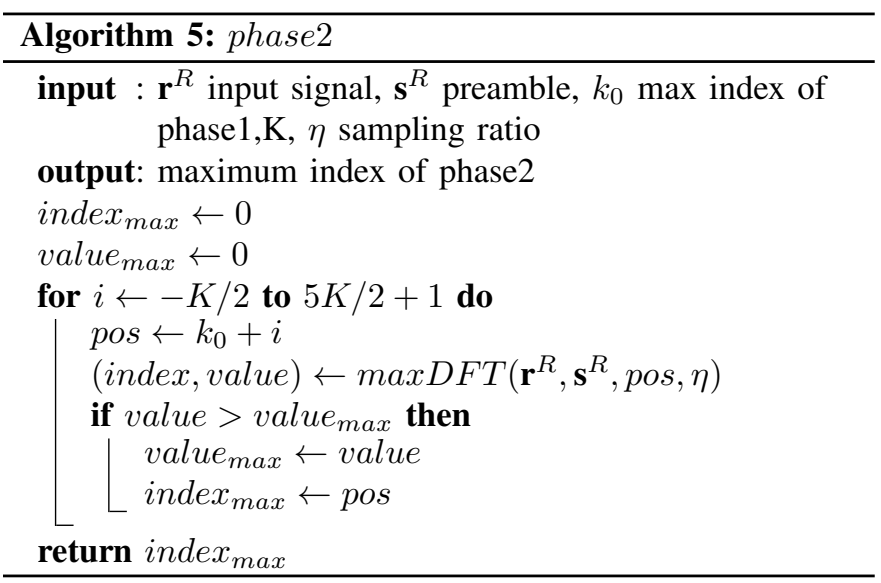

The second phase phase2 (see Algorithm 5) aims at searching the index $k_{1}$ of the local maximum of $g[k]$ the closest possible to $k_{0}$. In fact, we know from $Q[k]$ in (14) that $g$ oscillates and reaches local maxima every $K$ samples (with a default LoRa configuration of $K=8$ symbols preamble). Therefore, we search the index $k_{1}$ of the local maximum of (4) in the range $k_{0} \pm \frac{K}{2}$ samples. The complexity of Algorithm 5 is $O\left(\frac{(K+1) M}{\eta} \log \left(\frac{M}{\eta}\right)\right)$.

The third phase phase 3 (see Algorithm 6) searches the index $k_{2}$ of the maximum of $g$ around $k_{1}$. Given that $k^{*}$ is the index that maximize $g^{\prime}$ and $Q$, it should be located within $\llbracket k_{1}-u K, k_{1}+u K \rrbracket, u \in \mathbb{N}$, hence we search $k_{2}$ in

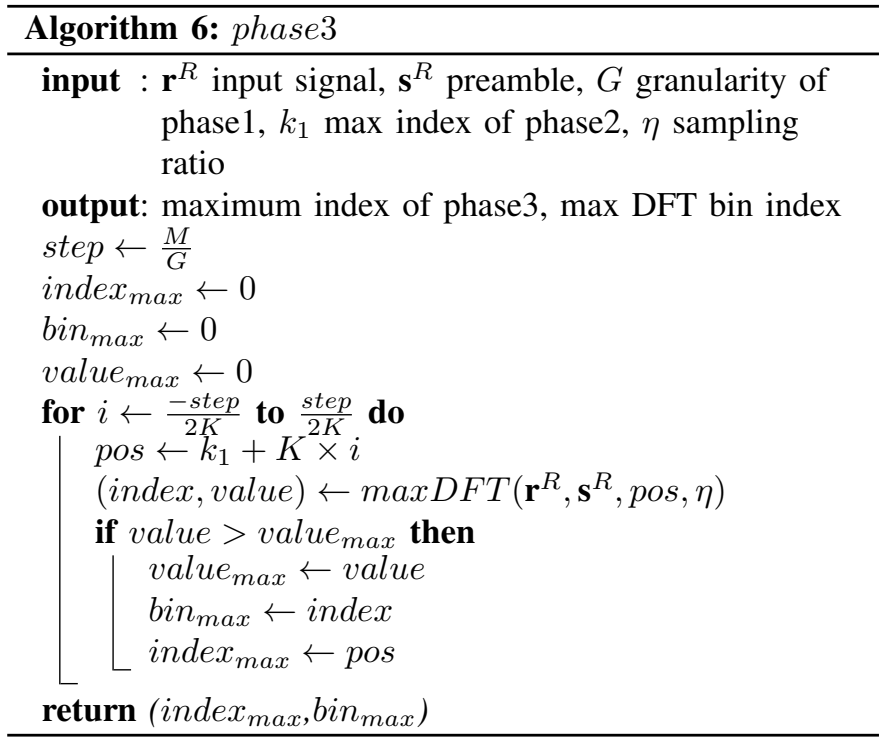

$k_{1} \pm u K$ with $u K \in \llbracket 0, \frac{M}{2 G} \rrbracket$. All the DFTs are performed using oversampling $R_{o}=2$ to further reduces the complexity which is about $O\left(\frac{M^{2}}{K \eta G} \log \left(\frac{M}{\eta}\right)\right)$ for this phase. It should be noted that this phase may be the heaviest in terms of calculation and in practice dominates the execution time. However, the number of calculations is widely reduced compared with the exhaustiveSynchro algorithm and makes the implementation usable in real condition including with SF12 while keeping a similar performance. Otherwise, the choice of $\mathrm{G}$ is a tradeoff between the cost of phase 1 and phase 3 , and empirically we find that $G=64$ gives the lowest execution times.

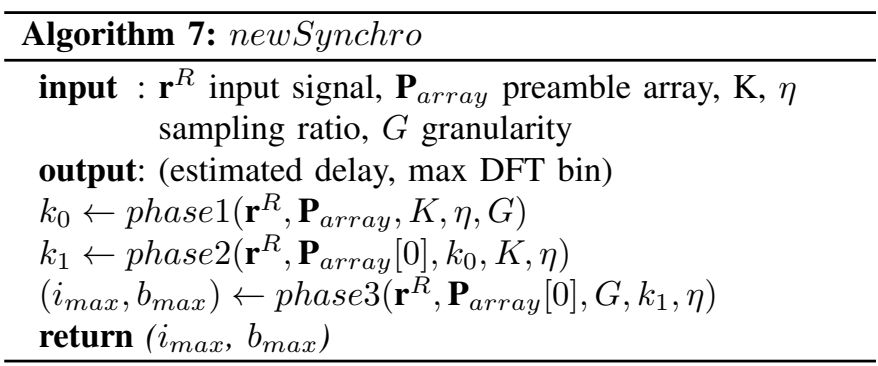

The overall algorithm is provided in the pseud-code of Algorithm 7. After estimating $k$ using the 3 previous phases, it also returns the position $b_{\max }$ of the DFT bin of the max of phase 3 , then the estimated frequency offset can be calculated thanks to $\hat{\theta}_{f}=b_{\max } \cdot \frac{N_{R}}{M T_{s}}$, where we remind that $\frac{N_{R}}{M T_{s}}$ is the frequency spacing between two bins.

\section{Simulations AND Discussion}

This section presents the simulations results, the complexity analysis, and a discussion of the suggested method and results. Simulations have been performed using Python 3 including numpy library, and the source code can be accessed at https: //github.com/b-com/synchro_LoRa. All results of Monte-Carlo simulations have been averaged over $10^{4}$ runs. We consider 


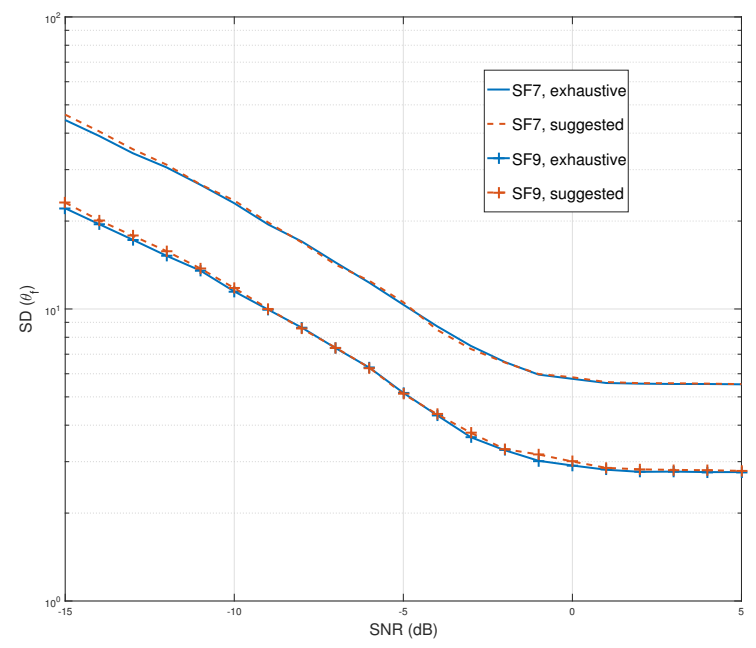

(a) $S D\left(\hat{\theta_{f}}\right)$ versus SNR $(\mathrm{dB})$.

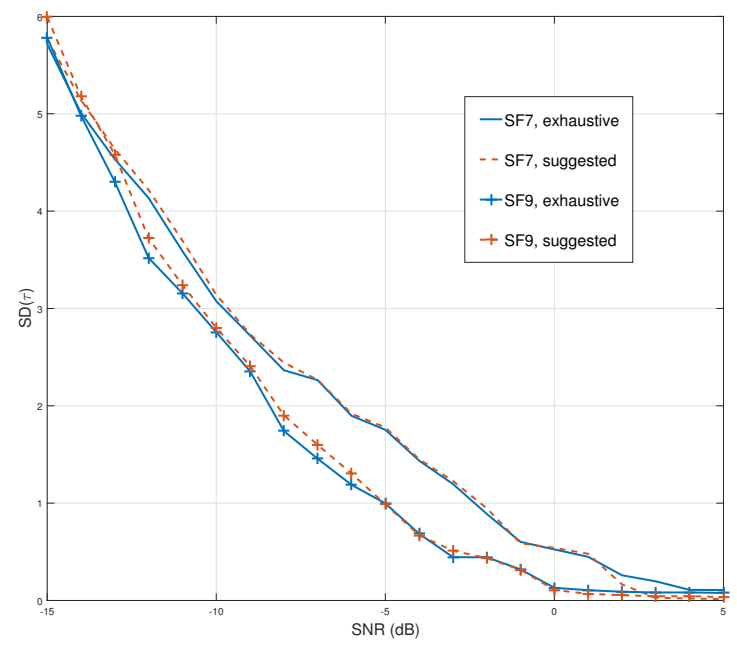

(b) $S D\left(\hat{n}_{\tau}\right)$ versus $\mathrm{SNR}(\mathrm{dB})$.

Fig. 6. SD performance of estimation versus SNR $(\mathrm{dB})$ for the frequency offset $\theta_{f}$ (a) and the delay $n_{\tau}$ (b). Comparison of the exhaustive search and the suggested algorithm for SF7 and SF9.

LoRa signals with SF7 and SF9, and with delays (in sampled version) and frequency offsets randomly chosen within the sets $n_{\tau} \in \llbracket 5,5+N_{R} \rrbracket$ and $\theta_{f} \in[-10,10] \mathrm{kHz}$, respectively. Moreover, we arbitrarily set $K=8, R=8, R_{0}=2$, and $G=64$, as we empirically found that this provides a good trade-off between performance and complexity.

\section{A. Simulations Results}

Fig. 6 shows the standard deviation of estimation of the frequency offset $\theta_{f}$ (Fig. 6-(a)) and the sampled delay $k$ (Fig. 6-(b)) versus SNR in the range $[-15,5] \mathrm{dB}$. The standard deviation (SD) of the estimate $\hat{X}$ of $X$, denoted by $S D$, is defined as

$$
S D(\hat{X})=\left(\frac{1}{N_{\text {run }}} \sum_{n=0}^{N_{\text {run }}}|\hat{X}-X|^{2}\right)^{1 / 2},
$$

where $N_{\text {run }}$ corresponds to the number of independent simulations runs. It can be observed in Fig. 6-(a) that the behavior of $S D\left(\hat{\theta}_{f}\right)$ of the suggested algorithm matches that of the exhaustive search, therefore validating the proposed implementation. Thus, we can notice that $S D\left(\hat{\theta}_{f}\right)$ decreases from $S N R=-15$ to $0 \mathrm{~dB}$, and reaches a lower bound for higher SNR values. This non-zero lower bound is due to the precision limitation inherent to the DFT, since the frequency spacing between two DFT bins is equal to $\frac{N_{R}}{M T_{s}}$. As a consequence, the absolute value of the error of estimation $\left|\hat{\theta}_{f}-\theta_{f}\right|$ falls within the interval $\left[0, \frac{N_{R}}{2 M T_{s}}\right]$. Moreover, it must be noted that $S D\left(\hat{\theta}_{f}\right)$ for SF9 is twice weaker than the SD for SF, e.g. $2.8 \mathrm{~Hz}$ for $\mathrm{SF} 9$ against $5.6 \mathrm{~Hz}$ for $\mathrm{SF} 7$ at $\mathrm{SNR}=5 \mathrm{~dB}$. This is consistent with the theory, as the frequency spacing between two bins is four times smaller in SF9 compared with SF7. Note that it has been proposed in [12], [14] to estimate the fractional frequency offset. This method could then be used in addition to the suggested low-complexity algorithm to further improve the estimator, but this is out of the scope of this paper.

We can observe in Fig. 6-(b) that the suggested technique and the exhaustive search have a similar behavior regarding the estimation of the delay. However, it can be noticed that the SD of SF9 coincides with that of SF7 in the SNR range lower than $-14 \mathrm{~dB}$, whereas SF9 outperforms SF7 for higher SNR values. This can be explained by the fact that the delay is chosen in an interval that is proportional to the size of the symbols, i.e. proportional to $2^{S F}$. Thus, in very low SNR values, the noise preponderates over the signal, and it is more likely to erroneously estimate $k^{*}=n_{\tau}$ if $n_{\tau} \in \llbracket 5,5+2^{9} \rrbracket$ (in SF9) rather than $n_{\tau} \in \llbracket 5,5+2^{7} \rrbracket$ (in SF7), even though the energy of the signal in SF9 compensates this phenomenon. In higher SNR range, the SD of SF9 is smaller than that of SF7, and reaches a lower bound of about tenth a sample, inherently due to the residual error on the frequency offset, since frequency offset and delay are intertwined in (12) and (13).

While Fig. 6 shows that the suggested algorithm reaches the performance of the exhaustive search, it remains to investigate its gain in terms of complexity. According to the asymptotic complexity of the algorithms assessed in Section IV, and the aforementioned chosen parameters, we find that the complexity of the exhaustive search and of the proposed method are $O\left(2^{2 S F+10} \log \left(2^{S F+4}\right)\right)$ and $O\left(2^{2 S F+1} \log \left(2^{S F+4}\right)\right)$, respectively. Thus, our algorithm is less complex by a factor 512 compared to the exhaustive search, in terms of number of operations. To provide more explicit results regarding the complexity, Table V-A gives the execution time of the suggested algorithm and the SD averaged over 100 runs. The results are obtained on a desktop with an Intel Xeon E5-1620 v4 @ $3.50 \mathrm{GHz}$ processor with $16 \mathrm{~GB}$ memory, and optimized using a $\mathrm{C}++$ implementation. In the same context, the execution time of the exhaustive algorithm in SF7 is about $280 \mathrm{~ms}$, namely about 100 times longer than the proposed method, and this times quadratically grows as a function of the SF. Thus, we find an average computation time of $25.7 \times 10^{3} \mathrm{~ms}$ 
TABLE I

EXECUTION TIME OF THE ALGORITHM OVER 100 RUNS.

\begin{tabular}{|l|l|l|}
\hline SF & Mean (ms) & Standard deviation \\
\hline 7 & 7,05 & 2,40 \\
\hline 8 & 16,88 & 7,34 \\
\hline 9 & 30,23 & 2,01 \\
\hline 10 & 63,56 & 4,64 \\
\hline 11 & 176,06 & 4,63 \\
\hline 12 & 494,81 & 21,06 \\
\hline
\end{tabular}

in SF10. Therefore these results give the advantage to our solution compared with the exhaustive search. In particular, it can be noticed from Table V-A that the duration of the synchronization is in the order of $0.5 \mathrm{~s}$ in SF12, whereas the typical frame duration is at least $1 \mathrm{~s}$. The proposed method therefore allows the LoRa receiver to perform the synchronization and demodulation in real time for any SF.

\section{B. Discussion}

This paper deals with both theoretical and implementation aspects of the synchronization in LoRa. To go further, it is noteworthy that a real-time LoRa receiver including the suggested synchronization algorithm has been implemented, such as described in [17], [18]. Different alternative solutions to the proposed one could be investigated to take advantage of the concavity of $g^{\prime}$ and further improve the complexity of the synchronization method, such as a gradient descent. Moreover, such as aforementioned, the suggested algorithm could be combined with fractional frequency offset estimators such as described in [12], [14], [15], in particular by using the two symbols of the sync word and the downchirps. Alternatively, the fractional frequency offset could be estimated by maximizing the function $\mathcal{Q}$ according to $\theta_{f}$, which does not require the sync word. Another lead for further researches consists in adapting the analysis and the algorithm to LoRa signal sampled at Nyquist rate, namely $R=R_{0}=1$. In fact, in this case, it must be noted that the results (12) and (13) do not hold anymore as $p_{1}$ and $p_{2}$ are merged due to the aliasing. Thus, this paper paves the way for further works on synchronization in the LoRa system.

\section{CONCLUSION}

In this paper, we have investigated the challenging problem of the LoRa chirp waveform receiver time and frequency synchronization. Based on a thorough study of the oversampled received LoRa signal model including time delay and frequency offset, we have derived the ML estimator for these two parameters. We have shown that the time and frequency synchronization problem can be translated into an optimization problem, where however the cost function is not concave. Our proposed approach, from a detailed analysis of the cost function, relies on a quick search of a near optimal estimate of the time delay, avoiding local optimums, followed by a search for the optimum in the vicinity of the first estimate. This proposal greatly reduces the complexity that would be required with the exhaustive search. Our results have shown that the ML estimation performance is reached, with a reduced calculation time, which allows for a real time software implementation of the synchronization on commodity hardware.

\section{APPENDiX A}

PROOF OF (4)

Basics on ML estimation can be found in [19]. We here apply the general principles in [19] to the LoRa signal (3). The likelihood function of a complex Gaussian variable $X \sim \mathbb{C N}\left(0, \sigma^{2}\right)$ is given by $f_{X}(x)=C \exp \left(-\frac{|x|^{2}}{\sigma^{2}}\right)$, where $C$ is a normalization constant that does not need to be detailed. We define $\Pi_{M^{\prime}}$ as an observation window of size $M^{\prime}$ such as $M^{\prime} \geq M+n_{\tau}$, i.e. we ensure that $\Pi_{M^{\prime}}$ of size $M^{\prime}$ contains the whole expected window of size $M$ containing the preamble. Moreover, we suppose that the noise samples $w[n]$ within $\Pi_{M^{\prime}}$ are independent and identically distributed. Then, the maximization of the likelihood function corresponding to the observation $r[n]$ in (3) leads to the ML estimator that can be expressed and simplified as in (23) since the maximization is independent of $C,|r[\eta n]|$, and $|h|$. Furthermore, the term

$$
e^{2 j \pi \frac{p R_{0} \eta_{2} S F}{M^{\prime} T_{S}} \times T_{s}\left(\frac{\eta n}{N_{R}}-\frac{\tau}{T_{s}}\right)},
$$

in the first line of (23) corresponds to the sampled version of the contribution of the frequency offset. It must be highlighted that the terms $\eta n$ indicates that an oversampling $R_{0}$ is used to perform the estimator, whereas the term $k$ indicates that the sliding window is shifted according to the oversampling rate $R$ (finest time granularity).

By taking the logarithm of the product in (23) and noting that the maximization is independent of $\sigma^{2}$, we obtain the following ML estimator:

$$
\begin{aligned}
\left(\hat{k}^{*}, \hat{p}^{*}\right)= & \underset{k, p}{\arg \max } \Re\left\{e^{2 j \pi \frac{p R_{0} 2^{S F}}{M^{\prime}}\left(\frac{k}{N_{R}}+\frac{\tau}{T_{s}}\right)} \times\right. \\
& \left.\sum_{n=0}^{M^{\prime} / \eta-1} r[\eta n] h^{*} s[\eta n-k]^{*} e^{-2 j \pi \frac{p \eta n}{M^{\prime}}-j \psi}\right\} .
\end{aligned}
$$

It must be emphasized that (24) can be solved under the assumption of prior knowledge of the channel $h$ and the phase $\psi$. Alternatively, if unknown, both parameters could be estimated and substituted in (24) to obtain the so-called concentrated likelihood function. However, it would increase the computation cost of the estimator and these parameters are not necessary for the synchronization. As a consequence, this step can be avoided by considering the modulus $|$.$| or$ the square modulus $|.|^{2}$ instead of the real part $\Re\{$.$\} in (24),$ therefore leading to

$$
\left(\hat{k}^{*}, \hat{p}^{*}\right)=\underset{k, p}{\arg \max }\left|\sum_{n=0}^{M^{\prime} / \eta-1} r[\eta n] s[\eta n-k]^{*} e^{-2 j \pi \frac{p \eta n}{M^{\prime}}}\right|^{2} .
$$

The term "r[ $[\eta n] s[\eta n-k] "$ in (25) corresponds to a fixed observation $r[\eta n]$ multiplied by a sliding window containing the samples of the reference signal $s[\eta n]$. In practice, it is more usual to consider a fixed reference signal $s[\eta n]$ and a sliding window containing the observations $r[\eta n+k]$ as the received 


$$
\begin{aligned}
\left(\hat{k}^{*}, \hat{p}^{*}\right)= & \underset{k, p}{\arg \max } C^{M^{\prime} / \eta} \prod_{n=0}^{M^{\prime} / \eta-1} \exp \left(-\frac{1}{\sigma^{2}}\left|r[\eta n]-h s[\eta n-k] \exp \left(2 j \pi \frac{p R_{0} \eta 2^{S F}}{M^{\prime} T_{s}} \times T_{s}\left(\frac{\eta n-k}{N_{R}}-\frac{\tau}{T_{s}}\right)+j \psi\right)\right|^{2}\right) \\
= & \underset{k, p}{\arg \max } C^{M^{\prime} / \eta} \prod_{n=0}^{M^{\prime} / \eta-1} \exp \left(-\frac{1}{\sigma^{2}}|r[\eta n]|^{2}\right) \exp \left(-\frac{1}{\sigma^{2}}|h|^{2}\right) \\
& \times \exp \left(\frac{2}{\sigma^{2}} \Re\left\{r[\eta n] h^{*} s[\eta n-k]^{*} \exp \left(-2 j \pi \frac{p R_{0} \eta 2^{S F}}{M^{\prime}}\left(\frac{\eta n-k}{N_{R}}-\frac{\tau}{T_{s}}\right)-j \psi\right)\right\}\right) \\
= & \underset{k, p}{\arg \max } \prod_{n=0}^{M^{\prime} / \eta-1} \exp \left(\frac{2}{\sigma^{2}} \Re\left\{r[\eta n] h^{*} s[\eta n-k]^{*} e^{-2 j \pi \frac{p \eta n}{M^{\prime}}-j \psi} e^{2 j \pi \frac{p R_{0} 2^{S F}}{M^{\prime}}\left(\frac{k}{N_{R}}+\frac{\tau}{T_{s}}\right)}\right\}\right),
\end{aligned}
$$

signal is sampled. Then we can limit to an observation window of size $M$ and then rewrite (25) as

$$
\left(\hat{k}^{*}, \hat{p}^{*}\right)=\underset{k, p}{\arg \max }\left|\sum_{n=0}^{M / \eta-1} r[\eta n+k] s[\eta n]^{*} e^{-2 j \pi \frac{p \eta n}{M}}\right|^{2},
$$

where we notice that the sum in the modulus corresponds to the DFT of $r[\eta n+k] s[\eta n]^{*}$, which finally leads to (4) and concludes the proof.

\section{APPENDix B}

PROOF OF (8)

To analyze $g[k]=\max _{p}\left|Y_{k, p}\right|^{2}$, we develop $Y_{k, p}$ as:

$$
\begin{aligned}
Y_{k, p} & =\sum_{n=0}^{K N_{R} / \eta-1} r[\eta n+k] s[\eta n]^{*} e^{-2 j \pi \frac{p \eta n}{M}} \\
& =\sum_{i=0}^{K-1} \sum_{n=i N_{R} / \eta}^{(i+1) N_{R} / \eta-1} \tilde{r}_{i}[\eta n+k] s_{i}[\eta n]^{*} e^{-2 j \pi \frac{p \eta n}{M}} .
\end{aligned}
$$

For $i \in \llbracket 1, K-1 \rrbracket$ (the case $i=0$ will be dealt with afterwards) and under the assumption that $\tau<<T_{s}$ we have

$$
\begin{aligned}
Y_{k, p, i}= & \underbrace{\sum^{(i+1) N_{R} / \eta-1} \tilde{r}_{i}[\eta n+k] s_{i}[\eta n]^{*} e^{-2 j \pi \frac{p \eta n}{M}}}_{\sum_{n=i N_{R} / \eta}} \\
= & \underbrace{\sum_{n=i N_{R} / \eta} \sum_{\left.\left.N_{R}-k\right) / \eta\right\rceil-1}^{(2)} \tilde{r}_{i}[\eta n+k] s_{i}[\eta n]^{*} e^{-2 j \pi \frac{p \eta n}{M}}}_{Y_{k, p, i}^{(1)}} \\
& +\sum_{\sum_{k, p, i}}^{\sum_{(i+1) N_{R} / \eta-1}^{n_{n=\left\lceil\left(n_{\tau}+i N_{R}-k\right) / \eta\right\rceil}[\eta n+k] s_{i}[\eta n]^{*} e^{-2 j \pi \frac{p \eta n}{M}}}},
\end{aligned}
$$

where $Y_{k, p, i}^{(1)}$ and $Y_{k, p, i}^{(2)}$ correspond to the samples between $i T_{s}$ and $i T_{s}+\tau$ on one hand, and $i T_{s}+\tau$ and $(i+1) T_{s}$ on the other hand, such as illustrated in Fig. 3. Let us now develop in detail the term $Y_{k, p, i}^{(1)}$ considering $w[n]=0$ in (29), where

$$
\begin{aligned}
\phi_{1}(k, i)= & \frac{B_{w}}{2}\left(\frac{k^{2}}{N_{R}^{2}}+\frac{\tau^{2}}{T_{s}^{2}}-\frac{2 \tau k}{T_{s} N_{R}}\right. \\
& \left.+(2 i-1)\left(-\frac{k}{N_{R}}+\frac{\tau}{T_{s}}\right)\right)+\theta_{f}\left(\frac{k}{N_{R}}-\frac{\tau}{T_{s}}\right)
\end{aligned}
$$

and $\gamma_{1}$ in defined in (10). We can finally obtain $Y_{k, p, i}^{(1)}$ as

$$
\begin{aligned}
Y_{k, p, i}^{(1)}= & h e^{j \psi} e^{2 j \pi T_{s} \phi_{1}(k, i)} e^{2 j \pi \eta \gamma_{1}(k, p) i N_{R} / \eta} \\
& \times \frac{1-e^{2 j \pi \eta \gamma_{1}(k, p)\left\lceil\left(n_{\tau}-k\right) / \eta\right\rceil}}{1-e^{2 j \pi \eta \gamma_{1}(k, p)}} \\
= & h e^{j \psi} e^{2 j \pi T_{s} \phi_{1}(k, i)} e^{2 j \pi \eta \gamma_{1}(k, p) i N_{R} / \eta} \\
& \times e^{j \pi \eta \gamma_{1}(k, p)\left(\left\lceil\left(n_{\tau}-k\right) / \eta\right\rceil-1\right)} \\
& \times\left\lceil\left(n_{\tau}-k\right) / \eta\right\rceil \operatorname{asinc}_{\gamma_{1}}\left(\left\lceil\left(n_{\tau}-k\right) / \eta\right\rceil\right),
\end{aligned}
$$

where $\operatorname{asinc}_{\gamma_{1}}($.$) is the aliased sinc function defined in (9).$ Similarly to the previous developments, we find that $Y_{k, p, i}^{(2)}$ can be expressed as

$$
\begin{aligned}
Y_{k, p, i}^{(2)}= & h e^{j \psi} \exp \left(2 j \pi T_{s} \phi_{2}(k, i)\right) \\
& \times \sum_{n=\left\lceil\left(n_{\tau}+i N_{R}-k\right) / \eta\right\rceil}^{(i+1) N_{R} / \eta-1} \exp \left(2 j \pi \eta n \gamma_{2}(k, p)\right),
\end{aligned}
$$

where

$$
\begin{aligned}
\phi_{2}(k, i)= & \frac{B_{w}}{2}\left(\frac{k^{2}}{N_{R}^{2}}+\frac{\tau^{2}}{T_{s}^{2}}-\frac{2 \tau k}{T_{s} N_{R}}\right. \\
& \left.+(2 i+1)\left(-\frac{k}{N_{R}}+\frac{\tau}{T_{s}}\right)\right)+\theta_{f}\left(\frac{k}{N_{R}}-\frac{\tau}{T_{s}}\right)
\end{aligned}
$$

and $\gamma_{2}$ is given in (11), which eventually yields: 


$$
\begin{aligned}
Y_{k, p, i}^{(1)}=h e^{j \psi} \sum_{n=i N_{R} / \eta}^{\left\lceil\left(n_{\tau}+i N_{R}-k\right) / \eta\right\rceil-1} & \exp \left(2 j \pi \frac{B_{w} T_{s}}{2}\left[\left(\frac{(\eta n+k)}{N_{R}}-(2 i-1)-\frac{\tau}{T_{s}}\right)\left(\frac{\eta n+k}{N_{R}}-\frac{\tau}{T_{s}}\right)-\left(\frac{2 \eta n}{N_{R}}-(2 i+1)\right) \cdot \frac{\eta n}{N_{R}}\right]\right) \\
& \times \exp \left(2 j \pi \theta_{f} T_{s}\left(\frac{\eta n+k}{N_{R}}-\frac{\tau}{T_{s}}\right)+j \psi\right) \exp \left(-2 j \pi \frac{p \eta n}{M}\right) \\
= & h e^{j \psi} \exp \left(2 j \pi T_{s} \phi_{1}(k, i)\right) \sum_{n=i N_{R} / \eta}^{\left\lceil\left(n_{\tau}+i N_{R}-k\right) / \eta\right\rceil-1} \exp \left(2 j \pi \eta n \gamma_{1}(k, p)\right)
\end{aligned}
$$

$$
\begin{aligned}
Y_{k, p, i}^{(2)}= & h e^{j \psi} e^{2 j \pi T_{s} \phi_{2}(k, i)} e^{2 j \pi \eta \gamma_{2}(k, p)\left\lceil\left(n_{\tau}+i N_{R}-k\right) / \eta\right\rceil} \\
& \times e^{j \pi \eta \gamma_{2}(k, p)\left(N_{R} / \eta-\left\lceil\left(n_{\tau}-k\right) / \eta\right\rceil-1\right)} \\
& \times\left(N_{R} / \eta-\left\lceil\left(n_{\tau}-k\right) / \eta\right\rceil\right) \\
& \times \operatorname{asinc}\left(\gamma_{2}(k, p)\left(N_{R} / \eta-\left\lceil\left(n_{\tau}-k\right) / \eta\right\rceil\right)\right) \\
= & h e^{j \psi} e^{2 j \pi T_{s} \phi_{2}(k, i)} e^{2 j \pi \eta \gamma_{2}(k, p) i N_{R} / \eta} \\
& \times e^{j \pi \eta \gamma_{2}(k, p)\left(N_{R} / \eta-1\right)} \\
& \times\left(N_{R} / \eta-\left\lceil\left(n_{\tau}-k\right) / \eta\right\rceil\right) \\
& \times \operatorname{asinc}_{\gamma_{2}}\left(\left(N_{R} / \eta-\left\lceil\left(n_{\tau}-k\right) / \eta\right\rceil\right)\right) .
\end{aligned}
$$

where asinc $_{\gamma_{2}}$ is defined in (9). To tackle the case $i=0$ and simplify the developments, we assume that $\tau$ is sufficiently weak to assume that $Y_{k, p, 0}$ can be expressed as in $(28)^{1}$, i.e. $Y_{k, p, 0} \approx Y_{k, p, 0}^{(1)}+Y_{k, p, 0}^{(2)}$, such that (27) can be approximated as

$$
Y_{k, p}=\sum_{i=0}^{K-1} Y_{k, p, i}^{(1)}+Y_{k, p, i}^{(2)}
$$

Finally, by noticing that

$$
\begin{aligned}
e^{2 j \pi \eta \gamma_{1}(k, p) i N_{R} / \eta} & =e^{2 j \pi i B_{w} T_{s}} e^{2 j \pi \eta \gamma_{2}(k, p) i N_{R} / \eta} \\
& =e^{2 j \pi \eta \gamma_{2}(k, p) i N_{R} / \eta}
\end{aligned}
$$

and

$$
\phi_{2}(k, i)=\phi_{1}(k, i)+B_{w}\left(\frac{\tau}{T_{s}}-\frac{k}{N_{R}}\right),
$$

we can then substitute (31) and (34) into (28) and then into (27) and (35), and considering the modulus $\left|Y_{k, p}\right|$, we obtain (8), which concludes the proof.

\section{REFERENCES}

[1] G. A. Akpakwu, B. J. Silva, G. P. Hancke, and A. M. Abu-Mahfouz, "A Survey on 5G Networks for the Internet of Things: Communication Technologies and Challenges," IEEE Access, vol. 6, no. 12, pp. 3619 3647, December 2017.

[2] W. Ayoub, A. Samhat, F. Nouvel, M. Mroue, and J.-C. Prévotet, "Internet of Mobile Things: Overview of LoRaWAN,DASH7, and NB-IoT in LPWANs standards andSupported Mobility," in proc. of ICT'18, June 2018, pp. $1561-1581$.

[3] K. Mekki, E. Bajic, F. Chaxel, and F. Meyer, "A comparative study of LPWAN technologies for large-scale IoT deployment," ICT Express, vol. 5, no. 1, pp. 1 - 7, March 2019.

${ }^{1}$ This assumption may be coarse for larger $\tau$ values, but it has no actual effect on the behavior of $g[k]$ in (14) due to the modulus. This justifies that the behavior analysis can be extended to any $\tau$ value.
[4] “Lorawan ${ }^{\mathrm{TM}}$ specification," LoRa Alliance Technical Committee, October 2017, ver. 1.1 .

[5] C. Gupta, T. Mumtaz, M. Zaman, and A. Papandreou-Suppappola, "Wideband chirp modulation for FH-CDMA wireless systems: coherent and non-coherent receiver structures," in proc. of ICC'03, Anchorage, AK, USA, May 2003, p. 2455 - 2459.

[6] L. Vangelista, "Frequency Shift Chirp Modulation: The LoRa Modulation," IEEE Signal Processing Letters, vol. 24, no. 12, pp. 1818 - 1821, December 2017.

[7] M. Chiani and A. Elzanaty, "On the LoRa Modulation for IoT: Waveform Properties and Spectral Analysis," IEEE Internet of Things Journal, vol. 6, no. 5, pp. 8463-8470, October 2019.

[8] T. Elshabrawy and J. Robert, "Closed-Form Approximation of LoRa Modulation BER Performance," IEEE Communications Letters, vol. 22, no. 9, pp. 1778 - 1781, September 2018.

[9] G. Ferré and A. Giremus, "LoRa Physical Layer Principle and Performance Analysis," in proc. of IEEE International Conference on Electronics, Circuits and Systems 2018 (ICECS'18). Bordeaux, France: IEEE, 12 2018, pp. $1-4$.

[10] C. F. Dias, E. R. de Lima, and G. Fraidenraich, "Bit Error Rate ClosedForm Expressions for LoRa Systems under Nakagami and Rice Fading Channels," Sensors, vol. 19, no. 20, pp. 1 - 11, October 2019.

[11] H. Tanaka, "A frequency and timing synchronization circuit making use of a chirp signal," EU Patent $99250131.2,1998$.

[12] M. Xhonneux, D. Bol, and J. Louveaux, "A Low-complexity Synchronization Scheme for LoRa End Nodes," ArXiv, no. arXiv:1912.11344, pp. 1 - 10, December 2019.

[13] R. Ghanaatian, O. Afisiadis, M. Cotting, and A. Burg, "Lora Digital Receiver Analysis and Implementation," in proc. of ICASSP'19, Brighton, United Kingdom, May 2019, pp. 1498-1502.

[14] C. Bernier, F. Dehmas, and N. Deparis, "Low Complexity LoRa Frame Synchronization for Ultra-Low Power Software-Defined Radios," IEEE Transactions on Communications, vol. 68, no. 5, pp. 3140-3152, May 2020.

[15] A. Marquet and N. Montavont, "Carrier and Symbol Synchronisation for LoRa Receivers," in proc. of EWSN'20, Lyon, France, February 2020, pp. $1-6$.

[16] O. Afisiadis, M. Cotting, A. Burg, and A. Balatsoukas-Stimming, "On the Error Rate of the LoRa Modulation with Interference," IEEE Transactions on Wireless Communications, vol. 19, no. 2, pp. $1292-$ 1304, February 2020.

[17] P. Savelli, V. Savaux, P. Desnos, A. Zeineddine, M. Kanj, and C. Delacourt, "Flexible Multi-standard Digital Front-End for LPWA Technologies," URSI Radio Science Letters, vol. 2, pp. 1 - 4, December 2020.

[18] C. Delacourt, P. Savelli, and V. Savaux, "A Cloud RAN Architecture for LoRa,” URSI Radio Science Letters, vol. 2, pp. 1 - 4, December 2020.

[19] S. M. Kay, Fundamentals of Statistical Signal Processing : Estimation Theory. Prentice Hall, 2003, ch. Maximum Likelihood Estimation, pp. $157-214$. 